\title{
Design of Integral Reminder for Collaborative Appointment Management
}

\author{
Ying Wang \\ University of Texas Rio Grande Valley \\ ying.wang01@utrgv.edu
}

\begin{abstract}
Reminder systems have great potential to enhance healthcare outcome, but there is a big space for improvement to facilitate the collaborative appointment management with accessible mobile communication technology. This study proposes a design of integral reminder systems that automates the process of appointment rescheduling for patients and physicians in addition to confirmation and cancellation. Based on the premises of patient-centered care of media synchronicity theory, design principles are proposed to cater to the different requirements of healthcare providers and patient users on appointment management. The design provides useful guidelines for building integral reminder systems that enhance medical compliance.
\end{abstract}

\section{Introduction}

Reminders are widely used in modern societies to reduce no-shows, missed deadlines to prior agreed appointments for events in an individual's personal, work and social lives. In healthcare, appointment reminder is important to reduce the high rate of nonattendance to healthcare appointments [24] [26] [37]. The appointment no-shows prevent patients from getting needed treatments and disrupt the operations of health clinics and practices, leading to serious economic and public health consequences [1] [17] [29].

There are different types of appointment reminder systems in healthcare which are marked by the communication media used. Reminders are delivered traditionally with postal mails and telephone calls, but email and cellular messaging are currently the most popular means [42]. With reminder systems based on such electronic channels, providers set up medical appointments with patients and send out emails and/or text messages to patients in advance to remind them of the upcoming appointments [6].

Not only are electronic reminder systems as effective as telephone reminders in reducing no-shows to medical appointments compared to no reminders, but also they are more cost-effective [24] [27]. However, existing reminder systems are designed as

\author{
Francis Andoh Baidoo \\ University of Texas Rio Grande Valley \\ francis.andohbaidoo@utrgv.edu
}

unidirectional message delivery or have very limited user interactivity. In the studies to evaluate the effectiveness of appointment reminder systems in healthcare, most systems send out reminder messages like the following: "You have an appointment with [clinic] on [date] at [time]. Please call [telephone number] ONLY if you cannot attend" [13] [16]. Some reminder systems may allow users to reply to the reminder messages with confirmation, but users cannot go beyond such low interactivity. When there is a schedule conflict, a user has to call the office to reschedule an appointment. Therefore, current reminder systems are limited in their capabilities to cater for user needs.

Rather than just receiving reminders passively, many contemporary patients want to play a more active role. If a user cannot make a scheduled visit, in particular, the person may reschedule by interacting with a reminder system without calling the office. This will make appointment management more convenient and flexible for patient users, as well as more cost effective for healthcare providers. This study proposes a design of reminder systems that facilitate integral process of appointment negotiation, including reminding, confirmation, cancellation, option giving and choice. Whereas such integral reminder systems may be used in many areas (e.g. library book return, bill payment, meeting arrangement), the discussion will focus on medical context as the potential health and economic impacts are huge.

\section{Research background}

\subsection{Patient-centered care}

Compared to traditional physician-centered medicine, the emerging patient-centered care reflects the trend to give intense consideration to patient needs and preferences in delivering healthcare services at present time [10]. Among the various definitions of patient-centered care, Berwick [3]'s is probably the most comprehensive and relevant to this study: "The experience (to the extent the informed, individual patient desires it) of transparency, individualization, recognition, respect, dignity, and choice in all matters, 
without exception, related to one's person, circumstances, and relationships in health care" (p. w560). This definition points out key issues related to patient needs in using medical appointment reminder system in a patient-centered care environment.

Existing information systems (e.g. email-based) used for appointment reminding simply push messages to patients who at most have the options to reply indicating either confirmation or cancellation [5] [7] [22] [33] [39]. New systems need to give patients more control and power for enhancing their participation and experience in patient-centered care. This study proposes a design of integral reminder systems that consider transparency, individualization, recognition, respect, dignity and choice. Rather than receiving reminders in a passive manner, patients are able to actively manage their own medical appointments through two-way negotiation with their providers.

In the investigation of the relationship between patient-centered care readiness and health digital inclusion, researchers suggested that the HIT used in patient-centered care has to cater to three basic needs of patient users: health information access, communication and coordination, and choice and empowerment [38]. In the case of appointment reminder systems, health information access pertains to the channels through which patients get informed of their appointment schedules, which may vary in accessibility and convenience depending on the media used. Communication and coordination require twoway interaction between patients and providers rather than one-way push of reminder messages. Choice and empowerment concern the content of interaction in terms of the scheduling options given to patients based on providers' availability.

Most current appointment reminder systems only allow unidirectional information access. That is, patients can only receive reminders of existing appointments and cannot access other related scheduling information. Even though some systems allow patients to confirm or cancel appointments, their ability to communicate and coordinate with the clinics on appointment scheduling and management is very limited. Users are not given any more choices in the process, and they are far away from getting empowered.

Integral reminder systems address the needs of patients in terms of access, coordination and choice by enabling two-way negotiation with providers on appointment management. For such a new class of systems, a sound design theory can provide essential guidelines on their implementation. Yet such a theory does not exist for integral reminder systems.

\subsection{Design science research}

During the last few decades, design science research (DSR) has evolved from its infancy to the current plethora. To guide the design of integral reminder systems in patient-centered care, it is necessary to develop a design theory based on the good understanding of user behavior in the new context. Among various design science approaches, the design theory approach is most appropriate for the following two reasons mentioned by Gregor and Iivari [19]. First, theorizing design knowledge provides a sound foundation for the rigor of DSR. Second, building design theories avoids re-invention of design artifacts and methods but leads to the accumulation of formal knowledge.

In a broad sense, a design theory involves the relationships among developers, artifacts, users and environment. More specifically, Walls et al. [41] defined information systems design theory (ISDT) as "a prescriptive theory which integrates normative and descriptive theories into design paths intended to produce more effective information systems" (p 36). Kernel theories adopted from natural/behavioral and social sciences are part of design theories as they provide the descriptive knowledge for the development of prescriptive design theory [41].

Walls, et al. [40] divided design theory into two major components: a design product and a design process. Both of them are built upon kernel theories and specify hypotheses and principles for designers to evaluate the IT artifact in question and its development process [40] [41]. The use of relevant IS theories is preferred to address the issues of IS serving as a reference discipline for others [2] and IS theories contributing to HIT research [35].

The development of a design theory focuses on the specification of general system requirements based on the understanding of the relationships among developers, clients and users [9]. In the case of integral reminder systems, the clients are healthcare providers who will install and operate the systems, but the direct end-users are patients. The design of such a system comprises two levels: general design regarding how it mediates and facilitates the collaboration between patients and providers, and specific design related to how it meets the requirement of user groups in mediated collaboration.

\section{An integral approach}

The gap between patient need for self-management of health-related schedules and the limited capabilities of current appointment reminder systems calls for a 
new design. The current systems are limited in patient participation: users only passively receive reminders with little ability to manage their own medical schedules. In this sense, such systems do not truly meet the requirement of patient-centered care since providers exercise full control over patients in the reminding process.

This study proposes a design of appointment reminder systems that enable patient users to actively manage schedules beyond just receiving alerts. A solution is to combine the functionalities of a reminder system and a scheduling system to enhance active schedule management for patients. This may reduce medical noncompliance, which is one of the primary factors that hinder effective disease control and prevention [4]. Patients missed scheduled activities mainly for two reasons: lapse of memory and conflict in schedule [30]. Current appointment reminder systems provide a solution to the first issue, but hardly address the second.

For instance, a patient user usually schedules a medical appointment a few weeks or months ahead of time, but may not be able to make it due to an unanticipated user priority event. When the user receives a one-way reminder under this circumstance, he/she can do little with it. The new design should give the patient rescheduling options. The patient is likely to choose an available slot, and avoid the total skipping of the appointment. By providing a "safe net" in schedule management, the integral approach is able to enhance patient compliance.

Providers also benefit from the new approach. First of all, it reduces no-shows that cause confusion and waste in terms of time and material set aside. Given other options, a patient is more likely to indicate the inability to come to an appointment rather than turning away from a reminder. Based on more accurate information of who will come and who will not, providers can manage schedules and associated resources more effectively. Also, the integration of scheduling and reminder systems may significantly release the burden for providers to manually communicate with patients and rearrange appointments.

Compared with existing approaches, the proposed integral approach allows patient users to go beyond access the scheduling system through the mediation of the reminder system. The process comprises of communication among three components: end-user device, reminder system and scheduling system. An end-user device can be a cellphone, smartphone, tablet, laptop or desktop, and the reminder system communicates with it in the form of emails or text messages. Such communication is based on the information from the scheduling system.
Physically, the scheduling system and reminder system can be separated on different computer servers or collocated on one machine. Nevertheless, they are functionally different: the scheduling system is basically a database server that handles schedule information, and the reminder system is a telecommunication server that directly interacts with end-user devices. The non-interactive approach only allows one-way communication from the reminder system to patient cellphones in the form of reminder messages. The existing interactive approach makes the front-end communication two-way by allowing patients to reply to reminder messages. However, the communication between the scheduling system and reminder system is still one-way. The integral approach further makes the back-end communication two-way, allowing patients to manage the data in the scheduling system through the reminder system.

\section{Kernel theory}

Electronic appointment reminding can be regarded as a computer-mediated communication between providers and patients. To better understand this type of communication, an examination of prior appointment reminders is helpful. Traditionally, providers mail or call patients to remind them of their upcoming appointments. Compared to direct face-toface communications, both postal and telephone reminders are delivered through mediated channels. In this sense, they are comparable to the appointment reminders delivered through digital networks, such as emails and SMS.

Media synchronicity theory can serve as a genuine IS kernel theory to addresses the synchronicity aspects of electronic media [11] [12]. According to the theory, media synchronicity is not a one-dimensional concept, but has multiple facets. The media capabilities that lead to different levels of media synchronicity can be classified into two general categories: transmission capabilities and processing capabilities [11]. Existing appointment reminder systems vary in the transmission and processing capabilities.

According to media synchronicity theory, media of different levels of synchronicity facilitate different types of communication processes in terms of conveyance and convergence. Conveyance refers to "the discussion of preprocessed information about each individual's interpretation of a situation, not the raw information itself", whereas convergence refers to "the transmission of a diversity of new information...to enable the receiver to create and revise a mental model of the situation" [11]. One major proposition of the media synchronicity theory is that communications for 
conveyance purposes usually require media of relatively low level of synchronicity, but communications for convergence purposes generally demand media of relatively high level of synchronicity [11].

\section{Meta requirements}

The integral reminder approach helps bridge the gap between healthcare providers and patient users on appointment management. On the one hand, providers want to minimize no-shows on scheduled appointments. On the other hand for whatever reasons, some clients may not be able to make the appointments and have to reschedule. Unidirectional reminders cannot satisfy user needs from both sides. Some emailand SMS-based reminder systems require patients to send back confirmation massages. Though the communication is two-way, a patient still has to call back to reschedule an appointment if the person cannot make it. What would be optimum for both provider and patient to manage appointment is the ability of the patient to access provider's appointment system and reschedule by selecting the most convenient available slot.

\subsection{Overall requirement}

In patient-centered care, patients need more control on the services related to their own healthcare by making informed decisions based on the options available [10]. In the process of appointment rescheduling through telephone, providers typically give patients some choices: for example, a provider may ask a patient whether he or she is available for the slots in the next few days; if the patient still gives negative answers, the provider will provide more options until they reach an agreement. However, this real-time two-way communication is time-consuming and costly as a provider can only handle one patient at a time.

An integral reminder system automates the negotiation process between providers and patients on appointment management. The overall requirement is automated dialogue: the new system is able to engage in a back-and-forth communication with a patient user without the need of actual human involvement. This approach is consistent with the premise of media naturalness theory that computer-mediated communication is likely to be smooth if it mimics human-human communication [23]. The existing applications, on the other hand, lack such a capability.

Iivari [21] summarized the seven archetypes of IT applications, and the following four utilitarian archetypes are particularly relevant to the interactive reminder system: 1) processor to automate; 2) medium to mediate; 3) tool to augment; and 4) information source to informate. These archetypes reflect the views of technology as labor substitution tool, productivity tool, social relations tool, and information processing tool respectively [31]. The overall requirement of automated dialogue is closely related to the first two archetypes of labor substitution and social mediation. That is, patients can negotiate appointment schedules through the reminder system without provider intervention. The next two archetypes are related to productivity and accessibility respectively. In patientcentered care, patient users want to make informed decision, and are mostly concerned with access to healthcare information directly related to them. On the other hand, provider users want to enhance the productivity so that they can serve more patients by minimizing cost and no-shows. Thus users and providers have different requirements specific to their own needs.

\subsection{Patient-side requirements}

An integral reminder system provides patients the information regarding existing appointments as well as the available time slots in case of schedule conflicts. With the information given, patients are able to confirm or reschedule appointments, and the system must allow a patient user to choose an available slot that works for the individual in the rescheduling process. As it is negation process in which the user is given options to choose from in order to reach an agreement, the first requirement of the new system from the patient perspective can be denoted as "negotiability". This requirement is mainly about patient access to alternatives: to reschedule an appointment, a patient wants to evaluate multiple options and choose the most convenient.

In addition to the content, another dimension of information accessibility is related to the time of retrieval and response. Recipients of electronic reminders expect quick and non-interruptive delivery of messages. For instance, email reminder users may not be aware of the messages until they log into their accounts. On the other hand, SMS reminder users usually get notified with the arrival of messages, but they do not have to read them until a convenient time later. When they have to reschedule appointments, there is also time to evaluate the options against their schedules, contrary to the telephone approach that typically requires them to respond right away. The second requirement from the patient perspective, therefore, can be denoted as "flexibility". 


\subsection{Provider-side requirements}

On the provider side, their concerns are not necessarily consistent with patients'. One of the primary concerns of providers is compliance, the extent to which preferred medical practices are followed [28]. In the context of medical appointments, patients need to come to clinics at the time scheduled based on their health conditions and previous treatments. Some situations are more restrictive than others, for example: the tuberculous (TB) skin test result should be checked within 72 hours. If a patient cannot make it to such an appointment, the reminder system should give alternative slots that are within the required time frame.

Based on this compliance requirement, providers are typically unwilling to offer patients too many choices. From the patients' perspective, however, they may prefer more choices based on the negotiability requirement. In this sense, the compliance requirement and negotiability requirement constitute two forces where the design of integral reminder systems has to strike a balance on the appropriate range of choices.

Similarly, patients usually want more time to evaluate the options given based on the flexibility requirement, but it is not the case for providers. When the integral reminder system gives a patient several alternative time slots, it will lock the slots so that other patients cannot take them. Providers do not like longlasting holds; rather, they want patients to make choices quickly so that more patients can get served. This requirement on the provider side may be termed as promptness, which locates on the other end of the time dimension from flexibility.

In summary, there are two dimensions of integral reminder system design that are related to the range of choices given and response time allowed respectively. Provider-side requirements and patient-side requirements are contrary to each other to different extent depending on particular situations. Therefore, the design of integral reminder systems may adapt to different situations in terms of the range of alternative choices and length of response time.

\section{Design principles}

Based on the kernel theory, the Meta requirements of integral reminder system can be translated into a set of design principles in terms of needed system capabilities. Appointment reminding process through media like mail and email can be regarded a communication process for conveyance purpose. That is, reminder messages convey the reminders from providers to patients; if a patient cannot make an appointment, the person need to call the office to reschedule. In comparison, telephone reminders may also serve the convergence purpose by allowing patients to discuss rescheduling options with providers directly. As expected, telephone has a relative high level of media synchronicity compared to mail and email.

Most existing appointment reminder systems (e.g. doctorconnect.net, 1call.com, clienttell.net, and voiceshot.com) are for the purpose of conveyance only as patients still have to call the office to reschedule appointments if needed. Even with interactive systems (e.g. talksoftonline.com), patients may only choose one of the two options, confirm or cancel, and cannot reach an agreement on rescheduling appointments with the use of the same media. Integral reminder systems, on the other hand, automatically facilitate the convergence process of appointment rescheduling that requires the communication and coordination of alternative schedules between providers and patients.

Principle \#1: Design to facilitate both conveyance and convergence processes between providers and patients

The first overall design principle of integral reminder systems is that they must support the convergence process between patients and providers to reach agreements on appointment rescheduling, in addition to the conveyance process of delivering reminders from providers to patients. This overall principle has implications on the implementation of more specific principles on system capabilities to guide the design of reminder systems such that they provide best interaction for users to manage appointment in real time. Between the two general types of media capabilities, transmission capabilities address the communication process between senders and receivers of messages, and processing capabilities address the encoding and decoding of messages at two ends [36]. Thus, transmission capabilities are closely related to the overall requirement of automated dialogue, and processing capabilities are closely related to patientand provider-side requirements.

Principle \#2: Design to have sufficient transmission capabilities for automated dialogue

Transmission capabilities include transmission velocity and parallelism, which pertain to the nature of electronic media. The selection of different media for reminder systems will lead to the variation in these two aspects. Media synchronicity theory proposes that media of higher synchronicity lead to better outcome if the communication process is for the purpose of convergence [11]. Transmission velocity is the basic requirement of synchronous communication, and thus the necessary condition for convergence communication process. In the case of appointment 
rescheduling, if messages take too much time to reach recipients, the options of available slots may not be valid anymore. Compared to transmission velocity, parallelism deals with how a medium can handle multiparty and two-way communications.

Based on the appointment schedule, a system may send out reminders within a certain period of lead time (e.g. 48 hours before the appointment time) automatically. When a patient receives a reminder message, the person may confirm or cancel an appointment. In case it is cancellation, the system will initiate the rescheduling process that involves multiple rounds of message exchanges with the patient just like the negotiation between a patient and a provider. The parallelism capacity of the system enables it to automatically handle the interactions with multiple patients at the same time, without the need for them to call back and the manual interventions of providers to reschedule appointments.

Whereas the transmission capabilities are genetic to the characteristics of electronic media, processing capabilities depend on system implementation adapted to the requirements of each user group. The major user groups of the integral reminder system in healthcare are providers and patients, who have different requirements. Patients have the negotiability and flexibility requirements, and providers have the compliance and promptness requirements. They imply different processing capabilities at each end, as the third principle suggests. Corresponding to each requirement, there is a more specific design principle on the relevant system capability.

Principle \#3: Design to adapt processing capabilities to the requirements of each user group

Negotiability suggests that patients be able to reach an agreement with providers on new appointments when there is a need to reschedule the existing ones. This is the key feature of integral reminder system in patient-centered care. It requires patients be given multiple options so that they can evaluate and select the best option that meets their schedules. The processing capability associated with this requirement is reprocessability that concerns the decoding of messages at the patient side [11]. Therefore, the design principle related to the patient-side reprocessability is that it must meet the negotiability requirement.

Principle \#3a: The patient-side reprocessability to meet the negotiability requirement

In the design of integral reminder systems, the messages must contain enough but not overwhelming information for patients to evaluate and make choices. If users are given too many options, they may incur excessive cognitive burden. Therefore, each message should contain a few options that are most relevant to patients. For example, if a patient cannot come to an appointment, the first few options will be given with the available slots close to the original date and time. In that way, the user can easily compare the options to make a choice. If the patient does not find any of them suitable, further groups of options may be successively presented.

From the providers' point of view, the compliance requirement constrains the range of options as healthcare-related activities need to be scheduled according to patients' health conditions and medical procedures. If patients are given "limitless" rescheduling options, the compliance will be compromised. The encoding of messages, therefore, must place a certain limit on the options offered to ensure compliance. The processing capability associated with the encoding of messages is rehearsability [11], and here it concerns the generation of messages at the provider side. Thus the design principle related to the provider-side rehearsability is that it must meet the compliance requirement.

Principle \#3b: The provider-side rehearsability to meet the compliance requirement

The design of integral reminder systems needs to allow providers to adapt the way options are given to different medical procedures and patient conditions. Take TB skin test for instance, options must be given within 72-hour window. Regular check-ups, on the other hand, allow more leeway. For most appointments, the extensions of one to two weeks may be acceptable. In case a patient is still undecided given all the options, the system may send a message explaining the importance of compliance and ask patients to choose from the given options to the best of their ability. Of course, patients can always call the office directly to ask for manual handling of special circumstances.

In addition to negotiability, patients want to have a certain extent of flexibility in responding to the messages received. It suggests that patients be given a reasonable amount of time to read and evaluate the options given. This is important as it can be distractive and interruptive to read reminder messages when users are engaged in activities like driving and meeting. For patients, it is preferred that the options remain valid as long as possible so that they do not need to restart the negotiation process. The time allowed for encoding response messages is associated with the capability of rehearsability on the patient side. This leads to the design principle that patient-side rehearsability must meet the flexibility requirement.

Principle \#3c: The patient-side rehearsability to meet the flexibility requirement

On the provider side, it is preferred that the patients respond to messages quickly, especially regarding the rescheduling options. This is consistent with the 
promptness requirement to ensure that the available time slots will not be locked for an extensive period of time so that other patients may be able to access them. Therefore, when an integral reminder system generates messages that contain rescheduling options, it should indicate the length of time during which the options remain valid. This implies a design principle related to provider-side rehearsability in order to meet the promptness requirement.

Principle \#3d: The provider-side reprocessability to meet the promptness requirement

\section{Design artifacts}

Patient-centered care stresses the importance of service accessibility as Berwick's [3] definition contains the phrase “without exception”. Thus, system accessibility is a key issue for medical appointment reminder systems, which can be implemented with different technologies varying in their accessibility. The first decision to make in the design of actual integral reminder systems, therefore, is the choice of electronic media through which messages are delivered in both directions.

To receive telephone and email reminders, patients must subscribe to landline and/or Internet services. However, a large proportion of people do not have telephones and computers at home due to various factors such as income, age and skill [8]. On the other hand, the population penetration of mobile telecommunication technology is much higher: for instance, over $90 \%$ of adults in the USA have cell phones [32]. Some reminder systems require the installation of applets on expensive and sophisticated smartphones (e.g. Clienttel Smartphone Reminder Applet), but more than one third of patients use regular cellphones that do not support such mobile health applications [14]. On the other hand, basic cellphones support short message service (SMS), making an ideal mobile technology in healthcare to enhance digital inclusion in the era of patient-centered care [25]. Compared with telephone, postal and email channels, users can receive SMS messages anywhere and anytime without much physical constraints (e.g. close to a computer) and social interruptions (e.g. taking a call in a meeting).

Thus, SMS is a preferred technology in the development of personal health records applications that are accessible to most patients [34]. For appointment reminder systems, in particular, its use is conducive to aforementioned design principles. First of all, SMS allows one-to-one delivery of messages and two-way communication between senders and receivers [15]. Such capabilities enable both conveyance and convergence processes involved in automated dialogue between providers and patients (Principle \#1).

Furthermore, integral reminder systems must have sufficient transmission capabilities, including velocity and parallelism (Principle \#2). Compared with other electronic media, SMS has relatively high potential in each. In terms of velocity, text messages are delivered directly to cellphones within seconds, without possible delays due to Internet access, software launch and/or account login (e.g. emails). In terms of parallelism, an integral SMS-based reminder system automates the negotiations with multiple patients simultaneously, and is high on both multiplicity and interactivity.

An integral appointment reminder system comprises the hardware and software components of scheduling system and reminder system as shown in Figure 4. Providers use their computers and mobile devices to interact with the scheduling system through the Web Server to access the appointment information in the Database. Patients use their cellphones to interact with the reminder system through the GSM Modem (hardware device that sends and receives short text messages) and SMS Gateway (software that operates the modem).

The middleware connecting the scheduling system and reminder system through Database and SMS Gateway is the main software component that implements the essential integral reminder algorithms. The other software components including SMS Gateway (Kannel), Web Server (Apache) and Database (MySQL) as well as operating system (Debian) are all free yet powerful open-source packages. Together with the hardware including server machine and GSM Modem, they meet the overall Meta requirement of automated dialogue between providers and patients. In particular, the two-way information flow through all the components enables both conveyance and convergence processes in appointment negotiation (Design Principle \#1). Also, the use of powerful opensource components supports such automated dialogue with sufficient transmission capabilities (Design Principle \#2).

The development of an integral reminder system needs to program the middleware that handles user interactions in terms of message generation and reception. The detailed design depends on the specific settings in which the system will be used. In particular, the patient-side Meta requirements in terms of negotiability and flexibility as well as the provider-side Meta requirements in terms of compliance and promptness need to be considered. The Middleware can be implemented with a high-level programming language like Python. Such a general-purpose language is widely used for scripting to generate dynamic 
content based on database access. The middleware so implemented will generate reminder messages based on the information in the schedule database, and modify the data based on user feedback. Through the web server, providers can retrieve updated schedule information.

Patients directly communicate with the reminder system through user interface, and different architecture designs lead to different interface designs shown in Figures 1 through 3 . Non-interactive interface only displays a reminder message. Interactive interface gives users the options for confirmation or cancellation. Integral interface enables deeper usersystem dialogue that allows a patient to reschedule an appointment.

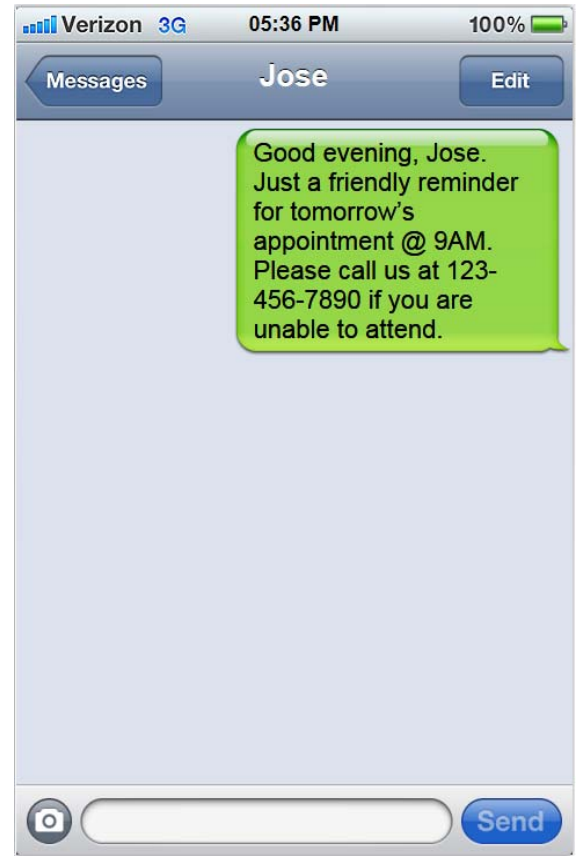

Figure 1. Non-Interactive Interface Design

The integral interface design adapts processing capabilities to the requirements of each user group (Design Principle \#3). In particular, a patient receives reasonable number of options to evaluate at a time in the process of appointment negotiation, which corresponds to Principle \#3a regarding patient-side reprocessability to meet negotiability requirement. The options compiled by the provider based on available slots are relatively close to the original appointment, which demonstrates Principle \#3b in terms of providerside rehearsability to meet compliance requirement. Once given the options, the patient does not have to respond right away but has some time to evaluate (e.g. check personal schedule) before making decisions, which pertains to Principle \#3c regarding patient-side rehearsability to meet flexibility requirement.
Meanwhile, the amount of time allowed is not excessive so that the provider can give optional slots to others in case of non-response, which follows Principle \#3d on provider-side reprocessability to meet promptness requirement.

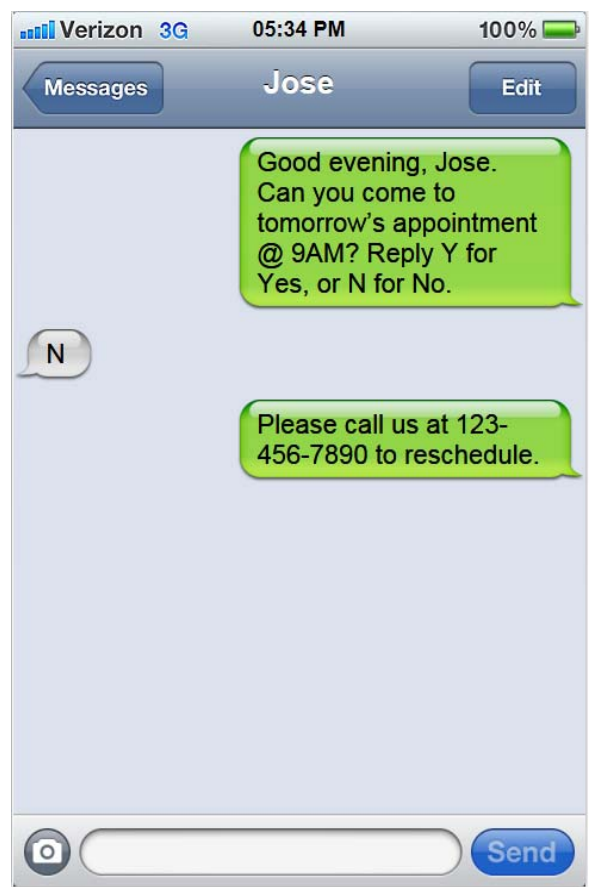

Figure 2. Interactive Interface Design

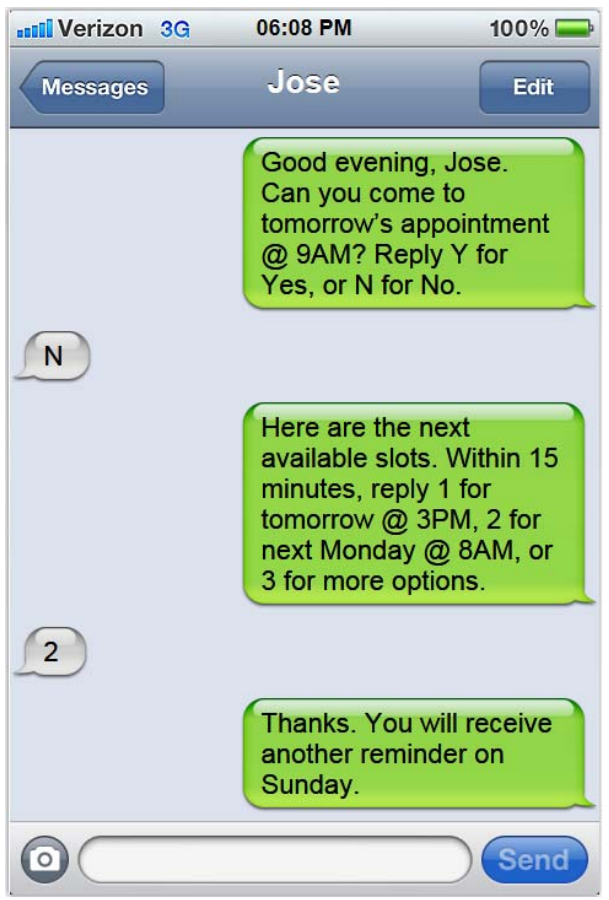

Figure 3. Integral Interface Design 


\section{Conclusion and Implications}

This study proposes a design theory of a new class of integral reminder systems in the context of patientcentered care based on the kernel theory of media synchronicity theory. The development of such a design theory integrates the understanding of user behavior, the design of technical artifacts and the context of healthcare practices. The Meta requirements were identified and the design principles were proposed for the implementation of integral reminder systems. Furthermore, general design methods and testable research hypotheses were also developed to provide some guidelines on future empirical studies.

The design theory may enhance patient participation in healthcare related to their medical appointments in the era of patient-centered care. Patient-centered care aims to improve the quality of healthcare services through the following seven aspects: 1) access to care; 2) patient engagement in care; 3) use of information technology to support care; 4) care coordination; 5) integrated care and smooth information transfer; 6) patient feedback; and 7) publicly available information on practices [12]. The proposed design theory can enhance all the seven aspects to some extent. Therefore, the design theory meets its goals to enhance patient experiences and health in medical services with the help of accessible and easy-to-use information technology.

The integral approach combines scheduling functionality with reminding functionality, and streamlines information processing in patient selfmanagement of medical schedules. The detailed designs can be adapted to various circumstances. For patients, such a system plays the role of "schedule advisor" rather than mere "alarming-clock". Assimilation of such a system will enhance the adherence of patients to medical appointments and interventions. For providers, the adoption allows them to keep track of medical scheduling and make informed decisions based on patient compliance. Thus the new approach is likely to have positive impacts on patient wellbeing, provider efficiency, and service effectiveness.

\section{References}

[1] A. Balikci, M. Erdem, S. Zincir, A. Bolu, S. B. Zincir, S. Ercan and O. Uzun, "Adherence with outpatient appointments and medication: A two-year prospective study of patients with schizophrenia", Bull Clin Psychopharmacol, 23, 2013, pp. 57-64.

[2] R. L. Baskerville and M. D. Myers, "Information systems as a reference discipline", MIS Quarterly, 26, 2002, pp. 1-14.
[3] D. M. Berwick, "What 'patient-centered'should mean: confessions of an extremist", Health Affairs, 28, 2009, pp. w555-w565.

[4] M. T. Brown and J. K. Bussell, Medication adherence: WHO cares? Mayo Clinic Proceedings, Elsevier, 2011, pp. 304-314.

[5] S. Can, T. Macfarlane and K. O'Brien, "The use of postal reminders to reduce non-attendance at an orthodontic clinic: a randomised controlled trial", British Dental Journal, 195, 2003, pp. 199-201.

[6] J. Car, I. Gurol-Urganci, T. de Jongh, V. VodopivecJamsek and R. Atun, "Mobile phone messaging reminders for attendance at healthcare appointments", The Cochrane Database Of Systematic Reviews, 12, 2013, CD007458.

[7] R. Chen, R. Sharman, N. Chakravarti, H. R. Rao and S. J. Upadhyaya, "Emergency response information system interoperability: development of chemical incident response data model", Journal of the Association for Information Systems, 9 (3), 2008, 7.

[8] M. D. Chinn and R. W. Fairlie, "The determinants of the global digital divide: a cross-country analysis of computer and internet penetration", Oxford Economic Papers, 2006.

[9] C. W. Churchman, The systems approach and its enemies, Basic Books New York, 1979.

[10] K. Davis, S. C. Schoenbaum and A. M. Audet, "A 2020 vision of patient-centered primary care", Journal of general internal medicine, 20, 2005, pp. 953-957.

[11] A. R. Dennis, R. M. Fuller and J. S. Valacich, "Media, tasks, and communication processes: A theory of media synchronicity", MIS quarterly, 32, 2008, pp. 575-600.

[12] A. R. Dennis and J. S. Valacich, Rethinking media richness: Towards a theory of media synchronicity, Systems Sciences, 1999. HICSS-32. Proceedings of the 32nd Annual Hawaii International Conference on, IEEE, 1999, pp. 10.

[13] S. R. Downer, J. G. Meara and A. C. Da Costa, "Use of SMS text messaging to improve outpatient attendance", Medical Journal of Australia, 183, 2005, pp. 366-368.

[14] G. Elliott, "The case for using SMS in mHealth", mhealthnews.com, 2013.

[15] B. S. Fjeldsoe, A. L. Marshall and Y. D. Miller, "Behavior change interventions delivered by mobile telephone short-message service", American journal of preventive medicine, 36, 2009, pp. 165-173.

[16] J. Foley and M. O’Neill, "Use of mobile telephone short message service (SMS) as a reminder: the effect on patient attendance", European Archives of Paediatric Dentistry, 10, 2009, pp. 15-18.

[17] A. George and G. Rubin, "Non-attendance in general practice: a systematic review and its implications for access to primary health care", Family Practice, 20, 2003, pp. 178184.

[18] S. Gregor and A. R. Hevner, "Positioning and presenting design science research for maximum impact", MIS Quarterly, 37, 2013, pp. 337-356.

[19] S. Gregor and J. Iivari, Designing for Mutability in Information Systems Artifacts, Canberra: ANU Press, 2007.

[20] A. R. Hevner, S. T. March, J. Park and S. Ram, "Design science in information systems research", MIS quarterly, 28, 2004, pp. 75-105. 
[21] J. Iivari, "A paradigmatic analysis of information systems as a design science", Scandinavian Journal of Information Systems, 19, 2007, pp. 39-64.

[22] M. L. Jibaja-Weiss, R. J. Volk, Q. W. Smith, J. D. Holcomb and P. M. Kingery, "Differential effects of messages for breast and cervical cancer screening", Journal of health care for the poor and underserved, 16, 2005, pp. 4252.

[23] N. Kock, P. Gray, R. Hoving, H. Klein, M. D. Myers and J. Rockart, "IS research relevance revisited: Subtle accomplishment, unfulfilled promise, or serial hypocrisy?", Communications of the Association for Information Systems, 8, 2002, pp. 23.

[24] E. Koshy, J. Car and A. Majeed, "Effectiveness of mobile-phone short message service (SMS) reminders for ophthalmology outpatient appointments: observational study", BMC ophthalmology, 8, 2008, pp. 1-6.

[25] S. Krishna, S. A. Boren and E. A. Balas, "Healthcare via cell phones: a systematic review", Telemedicine and eHealth, 15, 2009, pp. 231-240.

[26] G. Kunigiri, N. Gajebasia and D. Sallah, "Improving attendance in psychiatric outpatient clinics by using reminders", Journal of Telemedicine \& Telecare, 20, 2014, pp. 464-467.

[27] K. C. Leong, W. S. Chen, K. W. Leong, I. Mastura, O. Mimi, M. A. Sheikh, A. H. Zailinawati, C. J. Ng, K. L. Phua and C. L. Teng, "The use of text messaging to improve attendance in primary care: a randomized controlled trial", Family practice, 23, 2006, pp. 699-705.

[28] N. H. Miller, M. Hill, T. Kottke and I. S. Ockene, "The multilevel compliance challenge: Recommendations for a call to action A statement for healthcare professionals", Circulation, 95, 1997, pp. 1085-1090.

[29] C. G. Moore, P. Wilson-Witherspoon and J. C. Probst, "Time and money: effects of no-shows at a family practice residency clinic", Family Medicine-Kansas City-, 33, 2001, pp. 522-527.

[30] J. B. Norris, C. Kumar, S. Chand, H. Moskowitz, S. A. Shade and D. R. Willis, "An empirical investigation into factors affecting patient cancellations and no-shows at outpatient clinics", Decision Support Systems, 57, 2014, pp. 428-443.

[31] W. J. Orlikowski and C. S. Iacono, "Research commentary: Desperately seeking the "IT" in IT research-A call to theorizing the IT artifact", Information systems research, 12, 2001, pp. 121-134.

[32] PEW, Mobile Technology Fact Sheet: Highlights of the Pew Internet Project's Research Related to Mobile Technology, 2014.

[33] N. Roberts, K. Meade and M. Partridge, "The effect of telephone reminders on attendance in respiratory outpatient clinics", Journal of Health Services Research \& Policy, 12, 2007, pp. 69-72.

[34] D. W. Roblin, T. K. Houston, J. J. Allison, P. J. Joski and E. R. Becker, "Disparities in use of a personal health record in a managed care organization", Journal of the American Medical Informatics Association, 16, 2009, pp. 683-689.

[35] D. Romanow, S. Cho and D. Straub, "Editor's comments: riding the wave: past trends and future directions for health IT research", MIS Quarterly, 36, 2012, pp. III-A18. [36] C. E. Shannon and W. Weaver, The Mathematical Theory of Communication, University of Illinois Press, Champaign, IL, 1949.

[37] D. J. Sharp and W. Hamilton, "Non-attendance at general practices and outpatient clinics: Local systems are needed to address local problems", BMJ: British Medical Journal, 323, 2001, pp. 1081-1083.

[38] J. Sun, Y. Wang and N. Rodriguez, "Health digital inclusion and patient-centered care readiness in the USA", Communications of the Association for Information Systems, 32, 2013, Article 8.

[39] D. Thomas, "Postal reminders can improve attendance at orthodontic clinics", Evidence-based dentistry, 5, 2004, pp. 14-14.

[40] J. G. Walls, G. R. Widermeyer and O. A. El Sawy, "Assessing information system design theory in perspective: how useful was our 1992 initial rendition?", Journal of Information Technology Theory and Application, 6, 2004, pp. 43-58.

[41] J. G. Walls, G. R. Widmeyer and O. A. El Sawy, "Building an information system design theory for vigilant EIS", Information systems research, 3, 1992, pp. 36-59.

[42] J. Wei, I. Hollin and S. Kachnowski, "A review of the use of mobile phone text messaging in clinical and healthy behaviour interventions", Journal of Telemedicine and Telecare, 17, 2011, pp. 41-48. 